\title{
ADAPTIVE BEAMFORMING FOR HIGH BIT RATE SERVICES IN THE FDD MODE OF UTRA
}

\author{
Xavier Mestre, Montse Nájar, Carles Antón, Javier R. Fonollosa \\ Department of Signal Theory and Communications \\ Universitat Politècnica de Catalunya \\ c/ Jordi Girona 1-3, Mòdul D-5, Campus Nord UPC \\ 08034 Barcelona (Spain) \\ e-mail:\{mestre,najar,carles,fono\}@gps.tsc.upc.es
}

\begin{abstract}
Most time-reference beamforming algorithms suffer from severe beampattern distortion effects when applied to High Bit Rate services in WCDMA, causing serious performance degradation in terms of output BER, especially at high input SINR levels. These shortcomings are essentially caused by the uplink multiplexation of the traffic channel, which is seen by the base station as a powerful interfering source coming from the direction of arrival of the desired user.

In this paper, a semi-blind beamforming technique is proposed as a valid solution to overcome this effect. The suggested scheme resorts to a Conditional Maximum Likelihood approach to the underlying estimation problem and is designed to operate in an iterative fashion.
\end{abstract}

\section{INTRODUCTION}

The modulation format in the FDD mode of the UMTS Terrestrial Radio Access (UTRA) includes a set of training Pilot symbols code-multiplexed with the transmitted data stream. The (quasi)-orthogonality among spreading sequences allows the base station to use the pilot sequence either to obtain channel estimations or to perform spatial filtering.

This orthogonality between pilot and traffic channels is however not fully exploited when applying classical time-reference beamforming techniques. Traditionally, these techniques take no account of the temporal structure of the received signal (apart from the pilot sequence). As a consequence, the traffic channel is seen by the base station as a powerful interfering source coming from the direction of arrival of the desired user. Since beamformer weights are typically designed to null

This work was partially supported by the European Comission under ACTS Project AC347 SUNBEAM; the Spanish Government (CYCIT) TIC98-0412 TIC98-0703; and the Catalan Government (CIRIT) 1998SGR 00081.

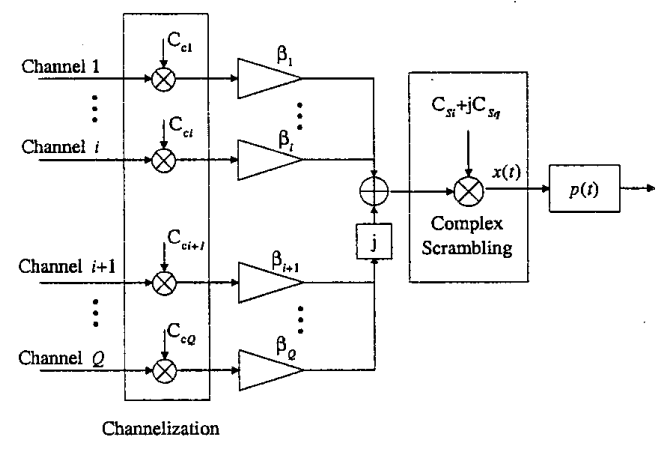

Figure 1: UTRA-FDD uplink modulation scheme.

out all undesired components of the received signal regardless of their direction of arrival, the spatial processing leads to a potential nulling (instead of an enhancement) of the desired user's contribution. This yields performance degradation in terms of output BER, especially at high SINR levels (see [5] for simulation results showing the consequences of this detrimental effect).

\section{SIGNAL MODEL}

Figure 1 depicts the basic modulation scheme used in the uplink of the FDD mode of UTRA. A particular user is assumed to generate $Q$ Dedicated Physical Channels, each one transmitted at a particular bit rate. One out of the $Q$ channels transports a pilot sequence, designed to allow channel estimation and/or space-time filtering at the reception stage.

The transmission of the different bit-rate streams over the physical channel is harmonized by means of Orthogonal Codes of variable length (OVSF codes) at the channelization stage. These Walsh-Hadamard sequences allow for the multi-rate structure of the signal, providing space for variable Spreading Factors $(S F)$ which may range from 256 (Low bit rate users) down to 4 (High bit rate users) chips/symbol. We denote $N_{c}$ the number of chips per slot, being a slot defined as one period of the 


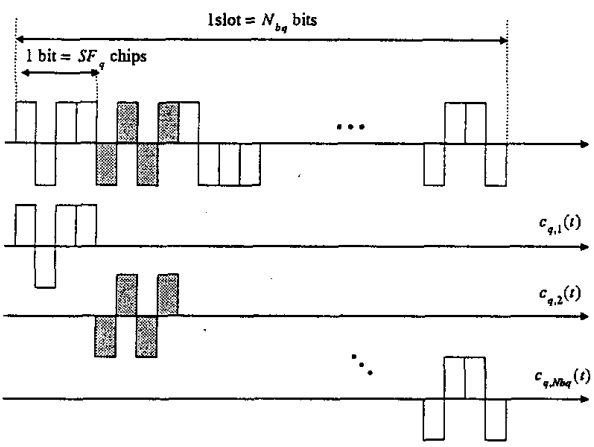

Figure 2: Slot configuration for the $q$-th channel

scrambling sequence. Only short scrambling codes -of length 256 chips - are considered here. The period of the channelization sequence for channel $q$ is set equal to $S F_{q}$, which is the spreading factor associated with that particular channel. With all the above definitions the number of bits per slot conveyed by this channel $\left(N_{b q}\right)$ can be expressed as $N_{b q}=\frac{N_{c}}{S F_{q}}$.

Finally, a series of power weights $\beta_{q}$ is designed to guarantee a proper quality of reception of all channels. The higher the bit rate, the more stringent the received Signal to Noise Ratio (SNR) requirements must be in order to preserve a similar received $\frac{E_{b}}{N_{0}}$. This means that in general high bit rate channels will have to be transmitted at higher power than low bit rate ones if similar raw BER requirements are assumed.

Since the harmful effects caused by the multiplexation of the traffic channels are especially noticeable at high received SNR, the solution proposed here will be particularly well suited to the reception of high bit rate users.

\subsection{TRANSMITTED SIGNAL}

Let $b_{q}(m)$ represent the underlying data stream associated with logical channel $q$. Its associated signal after the spreading operation has the following structure:

$$
x_{q}(t)=\sum_{s=-\infty}^{\infty} \sum_{i=1}^{N_{b q}} b_{q}\left(s N_{b q}+i\right) c_{q, i}\left(t-s N_{c} T_{c}\right)
$$

with $s$ the slot index, $T_{c}$ the chip period and $c_{q, i}(t)$ the complex signature corresponding to the $i$-th bit within a particular slot of the $q$-th logic channel stream. Notice that the scrambling stage causes a variation of the spreading sequence on a bit-interval basis. This is essentially equivalent to having a distinct spreading sequence for each symbol interval in a slot, conveniently zero-padded in order to maintain orthogonality with other time intervals (see Figure 2). Note that the complex signatures $c_{q, i}(t)$ as defined in (1) include the weight parameters $\beta_{q}$.
We can express both the bit sequence and the spreading signatures within a slot as $N_{b q} \times 1$ column vectors:

$$
\begin{aligned}
\mathbf{b}_{q}(s) & =\left[b_{q}\left(s N_{b q}+1\right) \cdots b_{q}\left(s N_{b q}+N_{b q}\right)\right]^{T} \\
\mathbf{c}_{q}(t) & =\left[c_{q, 1}(t), c_{q, 2}(t) \cdots c_{q, N_{b q}}(t)\right]^{T}
\end{aligned}
$$

Now, assuming that the mobile station transmits $Q$ distinct logical channels, we arrange all the transmitted data within slot $s$ and all the sequences used by the mobile station into larger column vectors:

$$
\begin{aligned}
\mathbf{b}(s) & =\left[\begin{array}{llll}
\mathbf{b}_{1}^{T}(s) & \mathbf{b}_{2}^{T}(s) & \cdots & \mathbf{b}_{Q}^{T}(s)
\end{array}\right]^{T} \\
\mathbf{c}(t) & =\left[\begin{array}{llll}
\mathbf{c}_{1}^{T}(t) & \mathbf{c}_{2}^{T}(t) & \cdots & \mathbf{c}_{Q}^{T}(t)
\end{array}\right]^{T}
\end{aligned}
$$

which have dimensions $N_{b} \times 1$, being $N_{b}=\sum_{q=1}^{Q} N_{b q}$ the number of transmitted bits in a slot interval.

With the above definitions, the resulting signal prior to pulse shaping turns out to be:

$$
x(t)=\sum_{q=1}^{Q} x_{q}(t)=\sum_{s=-\infty}^{\infty} \mathbf{b}^{T}(s) \mathbf{c}\left(t-s N_{c} T_{c}\right)
$$

In practice, each slot will convey some data known to the receiver. We will assume without loss of generality that this pilot sequence is represented by the $N_{b k}$ first components of the data vector $\mathbf{b}(s)$. This way, vector $\mathbf{b}(s)$ may be decomposed into $N_{b k} \times 1$ known $\mathbf{b}_{k}(s)$ and $N_{b u} \times 1$ unknown $\mathbf{b}_{u}(s)$ data vectors such that:

$$
\mathbf{b}(s)=\left[\begin{array}{ll}
\mathbf{b}_{k}^{T}(s) & \mathbf{b}_{u}^{T}(s)
\end{array}\right]^{T}
$$

where $N_{b k}$ and $N_{b u}$ represent the number of pilot symbols and unknown data bits in a slot respectively. Furthermore, the transmitted signal may be also separated into its known and unknown components:

$$
\begin{aligned}
x(t)= & \sum_{s=-\infty}^{\infty} \mathbf{b}_{k}^{T}(s) \mathbf{c}_{k}\left(t-s N_{c} T_{c}\right)+ \\
& +\sum_{s=-\infty}^{\infty} \mathbf{b}_{u}^{T}(s) \mathbf{c}_{u}\left(t-s N_{c} T_{c}\right)
\end{aligned}
$$

being $\mathbf{c}_{k}(t)$ and $\mathbf{c}_{\boldsymbol{u}}(t)$ their associated $N_{b k} \times 1$ and $N_{b u} \times 1$ sequence vectors such that:

$$
\mathbf{c}(t)=\left[\begin{array}{ll}
\mathbf{c}_{k}^{T}(t) & \mathbf{c}_{u}^{T}(t)
\end{array}\right]^{T}
$$

\subsection{RECEIVED SIGNAL}

Let us now consider the reception of a W-CDMA signal with an antenna array of $P$ elements. We restrict the scope of the paper to the reception using a single narrowband beamformer, whose weights will hereinafter be denoted $\mathrm{w}=\left[\begin{array}{lll}w_{1} & \cdots & w_{P}\end{array}\right]^{T}$. Assuming that the signal is transmitted through a frequency-selective channel with impulse response of 
length $L$ chips, the complex envelope of the signal received by sensor $p$ sampled at the chip rate can be modeled as follows [7]:

$$
r_{p}\left(n T_{c}\right)=\sum_{l=1}^{L} h_{p}(l, n) x\left((n-l) T_{c}\right)+n_{p}\left(n T_{c}\right)
$$

being $n_{p}(t)$ the noise plus interference component received by sensor $p$ and $h_{p}(l, n) l=1 \ldots L$ the overall discrete time-varying channel impulse response including both transmission and reception filters. We consider the reception of a single slot of the transmitted signal. Grouping $N=N_{c}+L-1$ samples received by the $P$ antennas in a common $N \times P$ matrix $\mathbf{R}$ and assuming that the channel is slowly varying (i.e. $L T_{c}<<\frac{1}{B_{d}}$ with $B_{d}$ the Doppler bandwidth) so that $h_{p}(l, n) \simeq h_{p}(l) \forall n$ within a slot in terval, one can write:

$$
\mathbf{R}=\left[\begin{array}{ccc}
r_{1}\left(T_{c}\right) & \cdots & r_{P}\left(T_{c}\right) \\
\vdots & & \vdots \\
r_{1}\left(N T_{c}\right) & \cdots & r_{P}\left(N T_{c}\right)
\end{array}\right]=\mathbf{X H}+\mathbf{N}
$$

with $\mathbf{H}$ the two-dimensional $L \times P$ channel matrix, $\mathrm{N}$ the $N \times P$ matrix of spatial-temporal noise samples;

$\mathbf{H}=\left[\begin{array}{ccc}h_{1}(1) & \cdots & h_{P}(1) \\ \vdots & & \vdots \\ h_{1}(L) & \cdots & h_{P}(L)\end{array}\right] \mathbf{N}=\left[\begin{array}{ccc}n_{1}(1) & \cdots & n_{P}(1) \\ \vdots & & \vdots \\ n_{1}(N) & \cdots & n_{P}(N)\end{array}\right]$

and $\mathrm{X}$ the $N \times L$ convolution matrix associated with the transmitted signal sampled at the chip rate (we assume that perfect synchronization with the user of interest has been attained already):

$$
\mathbf{X}=\left[\begin{array}{cccc}
x(0) & 0 & \cdots & 0 \\
\vdots & x(0) & & \vdots \\
x\left(\left(N_{c}-1\right) T_{c}\right) & \vdots & \ddots & x(0) \\
0 & x\left(\left(N_{c}-1\right) T_{c}\right) & \vdots \\
\vdots & & 0 & x\left(\left(N_{c}-1\right) T_{c}\right)
\end{array}\right]
$$

The signal after spatial filtering can be expressed as:

$$
\mathbf{y}=\mathbf{R w}=\mathbf{X h}+\mathbf{n}
$$

being $\mathbf{h}=\mathbf{H w}$ and $\mathbf{n}=\mathbf{N w}$ the equivalent channel impulse response and the noise contribution after the spatial processing.

Let us concentrate on the form of matrix X. Recalling the structure of the transmitted signal in (4) we can express this convolution matrix as:

$$
\mathbf{X}=\mathbf{C}\left(\mathbf{b} \otimes \mathbf{I}_{L}\right)
$$

with $\mathbf{b} \equiv \mathbf{b}(0), \mathbf{I}_{L}$ representing the $L \times L$ identity matrix and $\mathbf{C}$ being defined as:

$$
\mathrm{C}=\left[\begin{array}{llll}
\mathbf{C}_{1} & \mathrm{C}_{2} & \cdots & \mathbf{C}_{Q}
\end{array}\right]
$$

Each $\mathbf{C}_{q}$ in turn consists of:

$$
\mathbf{C}_{q}=\left[\begin{array}{llll}
\mathbf{C}_{q, 1} & \mathbf{C}_{q, 2} & \cdots & \mathbf{C}_{q, N_{b q}}
\end{array}\right]
$$

where each matrix $\mathbf{C}_{q, i}$ is constructed as the convolution matrix corresponding to the $i$-th bit conveyed over the $q$-th logical channel:

$$
\mathbf{C}_{q, i}=\left[\begin{array}{cccc}
c_{q, i}\left(T_{c}\right) & 0 & \cdots & 0 \\
\vdots & c_{q, i}\left(T_{c}\right) & & \vdots \\
c_{q, i}\left(N_{c} T_{c}\right) & \vdots & \ddots & c_{q, i}\left(T_{c}\right) \\
0 & c_{q, i}\left(N_{c} T_{c}\right) & & \vdots \\
\vdots & 0 & \ddots & c_{q, i}\left(N_{c} T_{c}\right)
\end{array}\right]
$$

Noting that the transmitted signal can be separated into its known and unknown parts, we may write $\mathbf{X}=\mathbf{X}^{k}+\mathbf{X}^{u}$. Furthermore, in accordance with (13) each convolution matrix can be expressed as:

$$
\begin{aligned}
& \mathbf{X}^{k}=\mathbf{C}^{k}\left(\mathbf{b}_{k} \otimes \mathbf{I}_{L}\right) \\
& \mathbf{X}^{u}=\mathbf{C}^{u}\left(\mathbf{b}_{u} \otimes \mathbf{I}_{L}\right)
\end{aligned}
$$

where matrices $\mathbf{C}^{k}$ and $\mathbf{C}^{u}$ are blocks of $\mathbf{C}$, associated with the sequences conveying known and unknown data respectively, i.e.:

$$
\mathrm{C}=\left[\begin{array}{ll}
\mathrm{C}^{k} & \mathrm{C}^{u}
\end{array}\right]
$$

\section{ML CRITERION FOR JOINT BEAMFORMING AND CHANNEL ESTIMATION}

Our objective is to design a joint beamforming and channel estimation scheme robust to the presence of the unknown data sequence $b_{u}$. Since a proper scheme must make implicit use of both known and unknown data, a semi-blind estimation framework seems the most logical approach to the problem. The aim of semi-blind techniques is to improve the training sequence estimations by using the statistical structure of the information sequences transmitted over the traffic channels. Classically, this has been shown to produce lower Cramér-Rao bounds in typical channel estimation problems [1], but to our knowledge its potential implications to the spatial filtering have never been investigated so far. We will show that semi-blind techniques yield dramatic performance improvements in both beamforming and channel estimation problems when dealing with WCDMA-modulated signals, providing solutions which clearly outperform those based only on training based procedures.

From the wide spectrum of possible estimation methods, we focus on Maximum Likelihood (ML) Estimators since under certain conditions they produce asymptotically efficient estimates. In particular, we will follow the Conditional (or deterministic) approach (CML) [8], which regards all symbols 
as deterministic parameters irrespective of whether they are known or not.

According to the CML criterion, if we presume that the noise component $\mathbf{n}$ in (12) is Gaussiandistributed, the maximum likelihood cost function after the beamforming can be expressed as:

$$
f_{\mathbf{b}_{u}, \mathbf{h}}(\mathbf{y})=\frac{1}{\operatorname{det}\left(\pi \mathbf{R}_{n}\right)} e^{-(\mathbf{y}-\mathbf{X h})^{H} \mathbf{R}_{n}^{-1}(\mathbf{y}-\mathbf{X h})}
$$

where $\mathbf{R}_{n}$ is the temporal covariance matrix of noise plus interference after the spatial processing stage. Assuming that both noise and interferences can be considered white in the temporal domain, i.e. $\mathbf{R}_{n} \simeq$ $\sigma^{2} \mathbf{I}$, the cost function becomes a mean squared error measure:

$$
\eta_{C M L}=\|\mathbf{y}-\mathbf{X h}\|^{2}
$$

with $\mathbf{X}=\mathbf{C}\left(\mathbf{b} \otimes \mathbf{I}_{L}\right)$ as shown in (13).

Now, taking derivatives with respect to $\mathbf{h}^{*}$ and forcing the result to zero, a maximum likelihood estimate of the channel is obtained:

$$
\hat{\mathbf{h}}=\left(\mathbf{X}^{H} \mathbf{X}\right)^{-1} \mathbf{X}^{H} \mathbf{y}
$$

where $\mathbf{X}$ includes both known and unknown data.

In order to find estimates of the unknown data, we substitute the estimation of the channel back into the cost function:

$$
\eta_{C M L}^{\prime}=\mathrm{y}^{H} \mathrm{P}_{x}^{\perp} \mathrm{y}
$$

with $\mathbf{P}_{x}^{\perp}=\left[\mathbf{I}-\mathbf{X}\left(\mathbf{X}^{H} \mathbf{X}\right)^{-1} \mathbf{X}^{H}\right]$. At this point, one can minimize (22) with respect to $b_{u}$ in order to obtain its maximum likelihood estimate. It is shown in [6] that the solution has the following expression:

$$
\hat{\mathbf{b}}_{u}=\left[\operatorname{Re}\left[\left(\mathbf{C}_{h}^{u}\right)^{H} \mathbf{C}_{h}^{u}\right]\right]^{-1} \operatorname{Re}\left[\left(\mathbf{C}_{h}^{u}\right)^{H}\left(\mathbf{y}-\mathbf{X}^{k} \hat{\mathbf{h}}\right)\right]
$$

with

$$
\mathbf{C}_{h}^{u}=\mathbf{C}^{u}\left(\mathbf{I}_{N_{b u}} \otimes \hat{\mathbf{h}}\right)
$$

and $\left(\mathbf{y}-\mathbf{X}^{k} \hat{\mathbf{h}}\right)$ the unknown component of the received signal. This solution could also be obtained minimizing the original cost function (20) and fixing $\mathbf{h}=\hat{\mathbf{h}}$ thereafter. In any case, it is seen that a necessary condition for the existence of a valid solution of (23) is $N \geq N_{b u}$, that is, the number of incoming snapshots must be larger than the number of unknown symbols to be estimated. Whenever this condition is not met, matrix $\left(\mathrm{C}_{h}^{u}\right)^{H} \mathbf{C}_{h}^{u}$ will become ill-conditioned and the proposed solution for the unknown data will not be implementable any more. A possible solution might consist in considering a larger number of received snapshots $(N)$, with the consequent reduction of the estimation variance. However this will not only result in a higher computational burden, but also in an intrinsic bias of the channel estimation due to its inherent nonstationarity.
On the other hand, dimensions of matrix $\left(\mathbf{C}_{h}^{u}\right)^{H} \mathbf{C}_{h}^{u}$ in (23) $-N_{b u} \times N_{b u}$ - will be generally too large for a practical implementation of its matrix inversion. Thus, adaptive solutions will be preferred in order to obtain valid estimates of the unknown data (see [6]). Inversion will be possible when dealing with short observation windows (i.e. one slot, as considered here) and low number of multiplexed channels.

Let us now consider the spatial filter design. We take derivatives with respect to the beamformer weights $\mathbf{w}^{*}$ in the concentrated cost function (22). The ML estimate of the weight vector nulling out the resulting gradient can be calculated as the eigenvector associated with the minimum eigenvalue of $\operatorname{matrix} \mathbf{R}^{H} \hat{\mathbf{P}}_{x}^{\perp} \mathbf{R}$ :

$$
\left(\mathbf{R}^{H} \mathbf{P}_{x}^{\perp} \mathbf{R}\right) \hat{\mathbf{w}}=\mathbf{0}
$$

with $\hat{\mathbf{P}}_{x}^{\perp}=\mathrm{I}-\hat{\mathbf{X}}\left(\hat{\mathbf{X}}^{H} \hat{\mathbf{X}}\right)^{-1} \hat{\mathbf{X}}^{H}$ and:

$$
\hat{\mathbf{X}}=\mathbf{X}^{k}+\mathbf{C}^{u}\left(\hat{\mathbf{b}}_{u}(s) \otimes \mathbf{I}_{L}\right)
$$

a structured estimate of the convolution matrix associated with the global transmitted signal. Computation of an eigenvector may result a somewhat consuming task, but one may find a less expensive approximation to the solution making use of (21):

$$
\hat{\mathbf{w}}=\left(\mathbf{R}^{H} \mathbf{R}\right)^{-1} \mathbf{R}^{H} \hat{\mathbf{X}} \hat{\mathbf{h}}
$$

being $\hat{\mathbf{h}}$ a channel estimate.

All the previous estimates suggest an iterative estimation algorithm, similar to the classic Ghosh. blind ML data-channel estimator [3]. Departing from an initialization of both the unknown data $\mathbf{b}_{u}$ and the beamformer weights $\mathrm{w}$, the algorithm operates as follows:

1. Find an estimate of the channel $\mathbf{h}$ from (21).

2. Find the unknown data $b_{u}$ using equation (23) or an adaptive implementation thereof.

3. Recalculate $\mathbf{w}$ using its $\mathrm{ML}$ estimate in (27).

4. Return to step 1 and repeat until convergence.

It is finally worth noting that convergence to local (and not global) minima will depend on the initialization of the algorithm. Nevertheless, the implicit use of the training sequence will yield quite reliable initial values and, as a consequence, the algorithm will be likely to reach the global minimum.

\section{SIMULATIONS}

Simulations indicate that, in practice, approximately 5 iterations are sufficient to provide global convergence of the algorithm. This is illustrated 


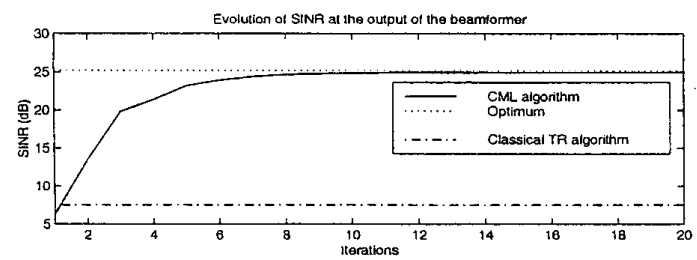

Evolution of the Channel Estimation MSE

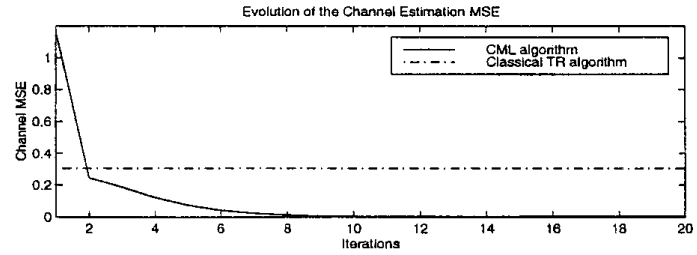

Figure 3: Evolution of channel estimation MSE and output SINR yielded by the semi-blind CML scheme vs. a classical Time Reference approach.

in Figure 3, where we have represented the beamformer output SINR and the channel estimation mean squared error (MSE) in a scenario with three mobile sources of equal power ( $20 \mathrm{~dB}$ above noise) transmitting two logical channels each $\left(S F_{q}=8\right.$, $\beta_{q}=1 \forall q$ ). The angular spread of the spatialtemporal mobile radio channel was set to 2 degrees, and its temporal dispersion was generated with a Vehicular model $(L=15)$ as specified by ETSI for UMTS [2]. Performance of the CML algorithm is compared against that of a classical Time Reference receiver scheme: the MDIR receiver [4]. In fact, the solution proposed by the MDIR receiver is no other than the one proposed here if explicit modeling of the unknown data is neglected.

The simulation shows how the inclusion of the unknown data estimation in the process of joint beamforming and channel estimation results in considerably higher output SINR and lower channel MSE. This would ultimately lead to a lower bit error rate at an output of an MLSE potentially placed after the spatial processing stage.

The second simulation illustrates the behavior of the two algorithms under distinct input SNR conditions (see Figure 4). The power of the three users present in the scenario ranged from -15 to $30 \mathrm{~dB}$ above the noise floor. The plot demonstrates how the proposed scheme is capable of achieving higher output SINR values (remarkably close to the optimum) and lower Channel MSE, especially at high values of the users' power.

\section{CONCLUSIONS}

A novel iterative scheme for WCDMA joint beamforming and channel estimation has been presented. The scheme is particularly intended for the adaptive antenna basestation operation in the FDD mode of UTRA, since it overcomes the potential auto-interference produced by the traffic channels.
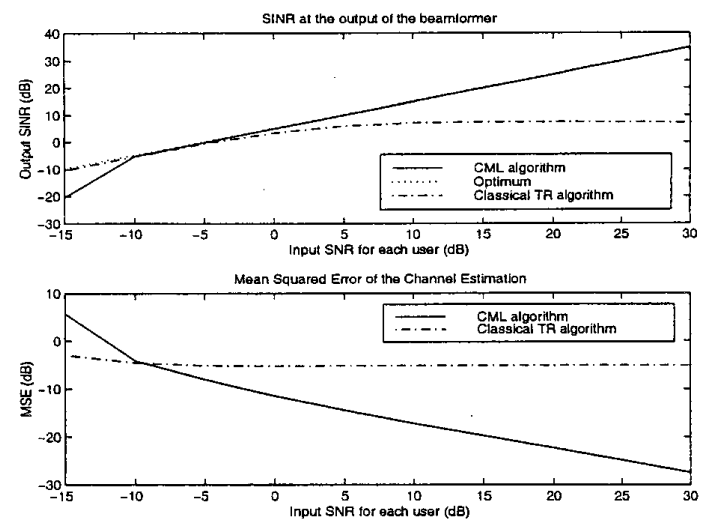

Figure 4: Steady state output SINR and channel estimation MSE as a function of the users' power with respect to the noise floor.

In contrast with other classical time-reference approaches relying uniquely on a known pilot sequence, the proposed framework makes implicit use of unknown data and signal structure to yield better performance results in both SINR and channel estimation MSE.

\section{REFERENCES}

[1] De Carvalho E., Slock D.T.M., "Cramer-Rao Bounds for Semi-blind, Blind and Training Sequence Based Channel Estimation", Proc. SPAWC'97, pp.129-132, Paris, April 1997.

[2] ETSI, "Selection Procedures for the Choice of Radio Transmission Technologies of the UMTS (UMTS 30.03)", Tech. Rep. TR101 112, v3.2.0, ETSI, 1998.

[3] Ghosh M., Weber C.L., "Maximum-likelihood blind equalization", Optical Engineering, vol. 36, no. 6, pp. 1224-1228, June 1992.

[4] M.A. Lagunas, A. Perez-Neira, J. Vidal, "Optimal Array Combiner for Sequence Detectors", Proc. ICASSP-98, vol IV, Seattle (WA), 1998.

[5] Mestre X., Fonollosa J.R., Vázquez G., "Uplink and Downlink Beamforming for the FDD mode of UTRA", Proc. IEEE VTC'99 (Spring), Houston, May 1999.

[6] Mestre X., Nájar M., Fonollosa J. R., "Joint spatial processing and channel estimation for high bit rate services in WCDMA", Submitted to IEEE Jounal on Selected Areas in Comm., Especial Issue on WCDMA (2nd Quarter 2000).

[7] Proakis J.G., Digital Communications, 3rd Edition, McGraw-Hill 1995.

[8] Stoica P., Nehorai A., "Performance Study of Conditional and Unconditional Directionof-Arrival Estimation", IEEE Transactions on $A S S P$, vol. 38, no. 10, October 1990. 